\title{
in
}

$X=641-73=21$

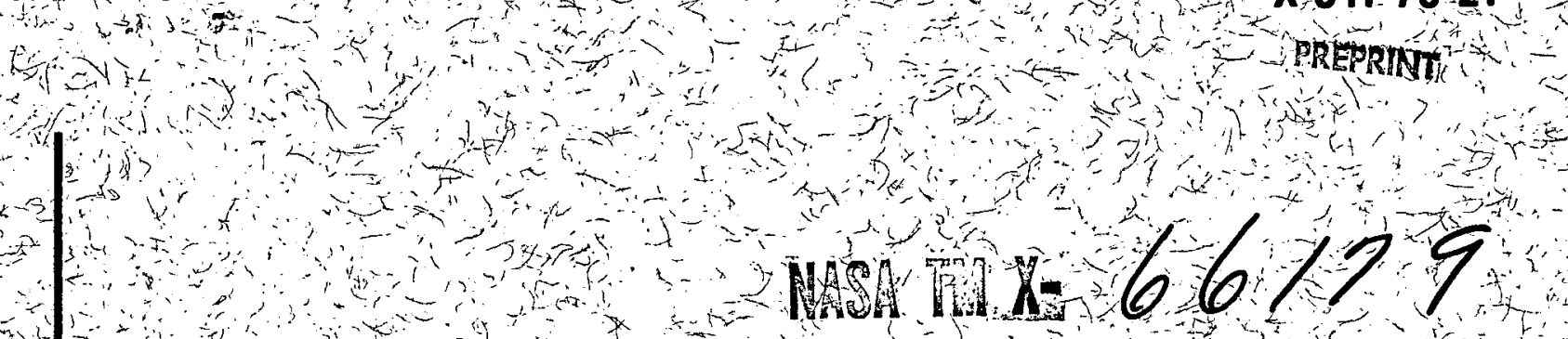

\section{POLARIZATION PHENOMENA IN MULTIPHOTON IONIZATION OF ATOMS}

V. JACOBS

(NASA-TH-X-66 179)

IN MULTIPHOTON ION POLARIZATION PHENOMENA

$27 \mathrm{p}$ HC $\$ 3.50$ : CSCL $20 \mathrm{H}$

N73-18718

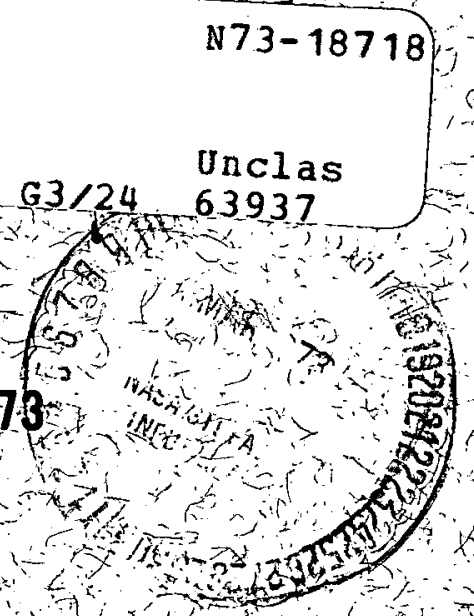

GSFC GODDARD SPACE FLIGHT CENTER GREENBELT, MARYLAND

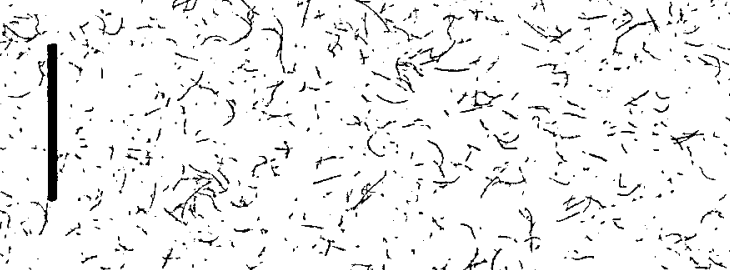


POLARIZATION PHENOMENA IN MUTPIPHOTON IONIZATION OF ATOMS

V. L. Jacobs*

Theoretical Studies Branch

Laboratory Ior Space Physics

Goddard Space Flight Center

National Aeronautics and Space Administration

Greenbelt, Maryland 20771

* Presently a National Academy of Sciences Research Associate working at Goddard Space Flight Center. 


\section{PRECEDING PAGE BLANK NOT FITMED}

Abstract

The theory of multiphoton ionization for an atomic system of arbitrary complexity is developed using a density matrix formalism. An expression is obtained which determines the differential $\mathbb{N}$-photon ionization cross section as a function of the polarization states of the target atom and the incident radiation. The parameters which characterize the photoelectron angular distribution are related to the general reduced matrix elements for the $\mathbb{N}$-photon transition. Two-photon ionization of unpolarized atoms is treated as an illustration of the use of the theory. The dependence of the multiphoton ionization cross section on the polarization state of the incident radiation, which has been observed in two- and three-photon ionization of Cs, is accounted for by the theory. Finally, the photoelectron spin polarization produced by the multiphoton ionization of unpolarized atoms, like the analogous polarization resulting from single-photon ionization, is Iound to depend on the circular polarization of the incident radiation.

\section{Preceding page blank}


1. Introduction

The experimental observations reported by Fox et al. (1971) and Kogan et al. (1971) indicate that the cross sections for two- and three-photon ionization of unpolarized cesium atoms depend on the polarization state of the incident radiation. Lambropoulos (1972a) has presented a theoretical interpretation of this effect in terms of the nonlinear dependence of the multiphoton transition amplitude on the photon polarization vector. Using a one-electron model for the atomic system, Lambropoulos (1972b) has shown that the differential N-photon ionization cross section for the case of circularly polarized light can be conveniently expressed in terms of only one dynamical coefficient. Because of the possibility that multiphoton ionization experiments will soon provide an important spectroscopic technique for probing atomic properties, it is desirable to formulate the theory without assuming any particular approximation for the atomic wave functions. In a previous paper (Jacobs 1972), the density matrix formalism (Fano 1957) was employed to develop a theory of single-photon ionization for an atomic system of arbitrary complexity. In this paper, the density matrix formalism is used to derive an expression which determines the differential N-photon ionization cross section as a function of the polarization states of the target atom and the incident radiation. The parameters which characterize the photoelectron angular distribution will be related to the general reduced matrix elements for the $\mathbb{N}$-photon transition. 
In the multiphoton photoelectric transition, the final continuum state of the ejected electron-residual ion system may be expanded in eigenstates of the total electronic angular momentum (partial waves). For incident photon frequency ranges up to and including the vacuum ultraviolet, the electric-dipole approximation should be adequate. The dipole selection rule implies that only a Iinite, and usually small, number of partial waves will be involved in the transition. This should be contrasted with the case of electron-atom scattering, where signiricant contributions to the cross section may be spread over a large number of partial waves. Providing that the range of tunable laser light sources can be extended into the vacuum ultraviolet, multiphoton ionization experiments could offer a precision technique of investigating the partial wave components of continuum atomic states.

In the Iormulation of the final continuum state partial wave expansion, it is customary to adopt the Is-representation when the effects of the spin-orbit interactions are not of interest. In this paper, particular emphasis will be given to the representation where the atomic states are specified by the total electronic angular momentum $J$ and the $z$-component $M$. The use of the J-representation is required when the fine structure of the atomic levels can be resolved in the photoionization experiment. The J-representation may still be employed even when the interactions can be assumed to be independent of $\mathrm{J}$, since the reduced matrix elements in the two alternative representations can then be related by means of vector coupling coefficients. The use 
of the J-representation will be essential for the correct determination of the photoelectron spin polarization produced by the interaction of heavy unpolarized alkali atoms with circularly polarized light (Fano 1969). An important aspect of this paper, which has not been exploited in previous treatments of multiphoton ionization, is the realization that the general form of the final state partial wave expansion can be written down without assuming any particular approximation for the atomic states beyond the choice of the angular momentum representation. Some investigations of multiphoton ionization have been concerned primarily with the phenomenon of laser-induced gas breakdown (Bebb and Gold 1966, Morton 1967). For the treatment of processes associated with very high light intensities, the perturbation treatment of the interaction between the atomic system and the radiation field is not valid; and other more suitable methods have been proposed (Reiss 1970, Faisal 1972). For the spectroscopic applications envisaged in this paper, the light intensity must be high enough to make the multiphoton transition observable. However, the light intensity should not be so high as to produce significant broadenings and shirts in the energy levels oI the atomic system under investigation. The perturbation treatment which is used in this paper should, therefore, be valid under the most favorable experimental conditions for spectroscopic studies.

Fox et al. (1972) have accounted for the observed photon polarization effect by noting that in the $\mathbb{N}$-photon ionization process the polarization of the incident radiation is partially transferred to the target atom 
during the first $N-I$ virtual transitions. ConsequentIy, in the N'th order virtual transition the interaction is between a polarized photon and a polarized atom. The density matrix formalism (Fano 1957) together with the spherical tensor operator formalism (Fano and Racah 1959), which are employed in this paper, lead to a convenient quantitative basis for this interpretation. A major portion oI this paper is devoted to deriving an expression which relates the spherical tensor components of the $\mathrm{I} i n a l$ state density matrix to the spherical tensor components of the target atom and the incident radiation polarizations. From this analysis it follows that in two-photon ionization of an Sstate of the target atom, the polarization of the first photon plays the same role as the target atom polarization in the case of singlephoton ionization from an aligned P-state. After making an appropriate reinterpretation of the reduced matrix elements, the form of the photoelectron angular distribution following multiphoton ionization can be deduced from the single-photon ionization formula with inclusion of the target atom polarization (Jacobs 1972 ).

The remainder of this paper is arranged as follows. In section 2 , the perturbation expression for the multiphoton transition operator is introduced. The expression for the spherical tensor components of the final state polarization density matrix is derived in section 3. Section 4 begins with a discussion of the form of the photoelectron angular distribution for the case where the ejected electron and the residual ion polarizations are not observed. As an illustration of the use of the theory, two-photon ionization of randomly oriented atoms is treated in both the IS- and the J-representations. Finally, the 
photolectron spin polarization produced by circularly polarized light is discussed.

\section{The Multiphoton Transition}

An example of the multiphoton transition considered in this paper is the simultaneous two-photon ionization process

$$
\operatorname{Cs}\left(1^{2} S_{1 / 2}\right)+2 \hbar \omega \rightarrow \mathrm{Cs}^{+}\left(1^{1} S_{0}\right)+e^{-}
$$

which has been observed by Kogan et al. (1971) by means of a pulsed ruby laser. For a general description of the multiphoton transition, the atomic states will be specified by the complete set of quantum numbers $\mathrm{n} J \mathrm{~J}_{\bullet}$. The symbol $\mathrm{n}$ is used to represent all quantum numbers which do not refer to angular momentum, such as the principle quantum number and the parity. In the case of light atoms it is usually more appropriate to use the specification NLS ML MS.

It will be assumed that the target atom (in the quantum state i) is singly ionized as a result of the simultaneous absorption of $\mathbb{N}$ identical electric-dipole photons with frequency $\omega$ and polarization vector $\vec{\epsilon}_{\lambda}$. The photoelectron is ejected with momentum $\vec{k}_{a}$ (corresponding to the formation of the residual ionic state a) and spin projection $m_{s}$. The magnitude of $\vec{k}_{a}$ is determined by the energy conservation relation

$$
E_{i}+N \omega=E_{a}+k_{a}^{2},
$$

which is expressed in rydbergs. It will be convenient to refer all 
projection quantum numbers, including $\lambda$ and $m_{s}$, to a common atomic quantization axis. The spin orientation $\overline{\mathrm{m}}_{\mathrm{S}}$, measured with respect to an arbitrary direction, may be obtained by employing the appropriate Wigner rotation matrix.

The polarization phenomena associated with the $\mathbb{N}$-photon ionization process may be conveniently investigated by introducing a transition operator $\underline{\mathrm{S}}^{(\mathrm{N})}$ whose matrix elements are given in lowest-order perturbation theory (Bebb and Gold 1966) by

$$
\begin{aligned}
& \left\langle M_{a} m_{s}\left|\underline{S}^{(N)}\right| M_{i} \lambda \ldots \lambda\right\rangle=2 \pi \sqrt{a \omega} \\
& \left\langle\cdot n_{a} J_{a} M_{a}, \vec{k}_{a} m_{s}\right|\left[\prod_{v=1}^{N-1} \sum_{n(v) J(v) M(v)} \frac{\vec{\epsilon}_{\lambda_{v+1}} \cdot \vec{Q}^{(1)}\left|n^{(v)} J^{(v)} M^{(v)}\right\rangle\left\langle n^{(v)} J^{(v)} M^{(v)}\right.}{E\left(n^{(v)} J^{(v)}\right)-E\left(n_{i} J_{i}\right)-v \omega}\right] \\
& \vec{\epsilon}_{\lambda_{1}} \cdot \vec{Q}^{(1)}\left|n_{i} J_{i} M_{i}\right\rangle,
\end{aligned}
$$

where $a$ is the fine structure constant, $\vec{Q}^{(1)}$ is the electric-dipole moment operator, and the virtual intermediate atomic states (including the continuum states) are specified by the quantum numbers $n{ }^{(v)}{ }_{J}(v){ }_{M}(v)$, $v=1, \mathbb{N}-1$. If the final continuum state is normalized per unit rydberg energy interval, the differential cross section for the transition where the four projection quantum numbers have definite values is given by

$$
\sigma_{a}^{(N)}\left(M_{a} m_{s} M_{i} \lambda, \vec{k}_{a}\right)=(2 \pi a \omega F)^{N-1}\left|\left\langle M_{a} m_{s}\left|\underline{S}^{(N)}\right| M_{i} \lambda \ldots \lambda\right\rangle\right|^{2} a_{0}^{2},
$$


where $F$ is the incident photon flux. The dependence on $F$ which appears in equation (4) implies that the order $\mathbb{N}$ of the multiphoton transition may be identified by varying the intensity of the incident radiation.

It is important to emphasize the simplifications which have been introduced into the perturbation expression (3). Firstly, it has been assumed that only one mode of the incident radiation field is excited. If the line-width of the light source is significant, it may be necessary to integrate equation (3) over the frequency distribution of the incident radiation. Even when the line-width of the light source can be neglected, a factor which can become as large as NI! should be included in equation (3) to account for the incoherence of the radiation. A further simplification is the omission of the radiative line-widths of the intermediate states from the energy denominators. For the resonant frequencies $\omega=\left[E\left(n^{(v)} J^{(v)}\right)-E\left(n_{i} J_{i}\right)\right] / v$, the radiative line-width must be included to give a finite denominator.

3. The Density Matrix Description

A more general description of the multiphoton ionization process may be achieved by the use of the density matrix formalism (Fano 1957). In this formalism, the polarization state of the target atom with definite angular momentum $\mathrm{J}_{i}$ is represented by $a\left(2 \mathrm{~J}_{\mathrm{i}}+1\right) \times\left(2 \mathrm{~J}_{\mathbf{i}}+1\right)$ density matrix $\underline{\rho}_{i}$. For the polarization state of the electric-dipole photon with frequency $\omega$, it will be convenient to employ the $3 \times 3$ density matrix $\underline{\rho}_{\mathbf{r}}$ which arises when the polarization vectors $\vec{\epsilon}_{\lambda}$ are referred to the atomic quantization axis. In general, the photon 
density matrix elements will depend on the orientation of the incident phot on beam with respect to the atomic quantization axis.

After introducing the direct product density matrix

$$
\underline{\rho}_{1}^{(N)}=\underline{\rho}_{i} \times \underline{\rho}_{r} \times \ldots \times \underline{\rho}_{r}
$$

where the photon density matrix $\rho_{\mathrm{r}}$ occurs $\mathbb{N}$ times, a complete analysis of the $\mathbb{N}$-photon ionization process may be given in terms of the Iinal state polarization density matrix

$$
\underline{\rho}_{2}^{(\mathrm{N})}=\underline{\mathrm{S}}^{(\mathrm{N})} \underline{\rho}_{1}^{(\mathrm{N})} \underline{\mathrm{S}}^{(\mathrm{N})+}
$$

that is

$$
\begin{aligned}
& \left\langle M_{a} m_{s}\left|\rho_{2}^{(N)}\right| M_{a}^{\prime} m_{s}^{\prime}\right\rangle=\sum_{M_{i} M_{i}^{\prime}} \sum_{\lambda_{1} \lambda_{i}^{\prime} \ldots \lambda_{N} \lambda_{N}^{\prime}}\left\langle M_{a} m_{s}\left|\underline{S}^{(N)}\right| M_{i} \lambda_{1} \ldots \lambda_{N}\right\rangle \\
& \left\langle M_{i} \lambda_{1} \ldots \lambda_{N}\left|\rho_{i}^{(N)}\right| M_{i}^{\prime} \lambda_{1}^{\prime} \ldots \lambda_{N}^{\prime}\right\rangle\left\langle M_{a}^{\prime} m_{s}^{\prime}\left|\underline{S}^{(N)}\right| M_{i}^{\prime} \lambda_{1}^{\prime} \ldots \lambda_{N}^{\prime}\right\rangle^{*} .
\end{aligned}
$$

The density matrix $\underline{\rho}_{2}^{(\mathrm{N})}$ determines the angular distribution and spin polarization of the ejected electrons together with the residual ion polarization as functions of the polarization states of the target atom and the incident radiation. If the initial state density matrices $\rho_{i}$ and $\rho_{-}$are normalized so that their traces are equal to unity, the diagonal elements of $\rho_{2}^{(\mathbb{N})}$ will be equal to the partial differential cross sections for the transitions to the $M_{a} m_{s}$ magnetic substates.

To represent the polarization density matrix Ior a system which is characterized by an angular momentum $\mathrm{J}$, it is convenient to introduce the spherical tensor operator $\mathrm{T}(\mathrm{K}, \mathrm{N})$ (Fano and Racah 1959, Happer 1968), which may be defined in terms of the Wigner 3-j symbol (the reference 
for the vector coupling coefficients used in this paper is de Shalit and Talmi 1963) by

$$
\left\langle\mathrm{J} M|\underline{T}(\mathrm{~K}, \mathrm{~N})| \mathrm{J} M^{\prime}\right\rangle=(-1)^{\mathrm{J}^{-} \mathrm{M}}(2 \mathrm{~K}+1)^{3 / 2}\left(\begin{array}{ccc}
\mathrm{J} & \mathrm{K} & \mathrm{J} \\
-\mathrm{M} & \mathrm{N} & \mathrm{M}^{\prime}
\end{array}\right)
$$

The density matrix $\underline{\rho}$ which describes the polarization state of the system may be expanded in terms of the complete set of $(2 \mathrm{~J}+1)^{2}$ spherical tensor operators in the form

$$
\underline{\rho}=\sum_{\mathbf{K N}} \rho(\mathbf{K}, \mathbf{N}) \underline{\mathbf{T}}(\mathbf{K}, \mathbf{N})
$$

The spherical tensor (or multipole polarization) components $\rho(K, N)$ are determined by the relation

$$
\rho(\mathrm{K}, \mathrm{N})=\left(\mathrm{tr} \underline{\rho} \underline{\mathrm{T}}(\mathrm{K}, \mathrm{N})^{+}\right)
$$

The initial state polarization density matrix $\rho_{1}^{(\mathrm{N})}$ defined by equation (5) may now be conveniently represented by the expansion

$$
\begin{aligned}
\rho_{1}^{(N)}= & \sum_{\mathbf{K}_{i} N_{i} K_{r}^{(1)} N_{r}^{(1)} \ldots K_{r}^{(N)} N_{r}^{(N)}} \rho_{i}\left(K_{i}, N_{i}\right) \underline{T}\left(K_{i}, N_{i}\right) \\
& \times \rho_{r}\left(K_{r}^{(1)}, N_{r}^{(1)}\right) \underline{T}\left(K_{r}^{(1)}, N_{r}^{(1)}\right) \times \ldots \times \rho_{r}\left(K_{r}^{(N)}, N_{r}^{(N)}\right) \underline{T}\left(K_{r}^{(N)}, N_{r}^{(N)}\right),
\end{aligned}
$$

where the spherical tensor operators $\underline{\underline{T}}\left(\mathrm{~K}_{i}, \mathbb{N}_{i}\right)$ and $\underline{\underline{T}}\left(\mathrm{~K}_{r}, \mathbb{N}_{r}\right)$ operate within the subspaces of the target atom angular momentum $J_{i}$ and the 
photon angular momentum $J_{r}=1$, respectively. Similarly, the final state polarization density matrix $\rho_{2}^{(\mathbb{N})}$ will be expanded in the form

$$
\begin{aligned}
\rho_{2}^{(N)}= & \sum_{\mathbf{K}_{a} N_{a}} \sum_{K_{s} N_{s}} \sum_{L M} \rho_{2}^{(N)}\left(K_{a} K_{s} L, N_{a} N_{s} M\right) \\
& \underline{T}\left(K_{a}, N_{a}\right) \underline{T}\left(K_{s}, N_{s}\right) Y_{L M}\left(\hat{K}_{a}\right),
\end{aligned}
$$

where $\underline{T}\left(K_{a}, N_{a}\right)$ corresponds to the residual ion with angular momentum $J_{a}, \underline{T}\left(K_{G}, \mathbb{N}_{\mathbb{S}}\right)$ corresponds to the ejected electron with angular momentum $s=I / 2$, and $Y_{I M}\left(\hat{k}_{a}\right)$ denotes the spherical harmonic function of the ejection angles.

To obtain the explicit expression for the tensor components of $\rho_{2}^{(N)}$, it will be necessary to introduce into equation (3) the rinal state partial wave expansion (Blatt and Biedenharn 1952, Jacobs 1972) with the incoming wave boundary condition appropriate to the description of the photoionization process (Breit and Bethe 1954). In the Jrepresentation this expansion may be written as

$$
\begin{aligned}
& \left|\mathrm{n}_{\mathrm{a}} \mathrm{J}_{\mathrm{a}} \mathrm{M}_{\mathrm{a}}, \overrightarrow{\mathrm{k}}_{\mathrm{a}} \mathrm{m}_{\mathrm{s}}\right\rangle=\sum_{\ell \mathrm{m}} \sum_{\mathrm{K} N} \sum_{\mathrm{J} M} \mathrm{i}^{\ell} \mathrm{e}^{-\mathrm{i} \sigma \ell\left(\mathrm{k}_{\mathrm{a}}\right)} \mathrm{Y}_{\ell_{\mathrm{m}}}^{*}\left(\hat{\mathrm{k}}_{\mathrm{a}}\right) \\
& (-1)^{\mathrm{s}-\mathrm{J}_{\mathrm{a}}-\mathrm{N}+\mathrm{K}-\ell-\mathrm{M}} \sqrt{(2 \mathrm{~K}+1)(2 \mathrm{~J}+1)}\left(\begin{array}{ccc}
\mathrm{J}_{\mathrm{a}} & \mathrm{s} & \mathrm{K} \\
\mathrm{M}_{\mathrm{a}} & \mathrm{m}_{\mathrm{s}} & -\mathrm{N}
\end{array}\right)\left(\begin{array}{ccc}
\ell & \mathrm{K} & \mathrm{J} \\
\mathrm{m} N & -\mathrm{M}
\end{array}\right)\left|\mathrm{n}_{\mathrm{a}} \mathrm{J}_{\mathrm{a}} \mathrm{K} \ell, J M\right\rangle,
\end{aligned}
$$

where $\sigma_{\ell}\left(k_{a}\right)$ is the Coulomb phase shift. The asymptotic form of the angular momentum eigenfunctions $\left|\mathrm{n}_{\mathbf{a}} \mathrm{J}_{\mathbf{a}} \mathrm{K} \ell, \mathrm{J} M\right\rangle$ for large distances between the ejected electron and the residual ion involves the scattering matrix for the electron-ion collision problem. 
The corresponding form of the partial wave expansion in the LS-representation is

$$
\begin{aligned}
& \left\lceil n_{a} L_{a} S_{a} M L_{a} M S_{a}, \vec{k}_{a} m_{s}\right\rangle=\sum_{\ell_{m}} \sum_{L M L} \sum_{S M S} i^{\ell} e^{-i \sigma l\left(k_{a}\right)} Y_{\ell_{m}^{*}}^{*}\left(\hat{k}_{a}\right) \\
& (-1)^{\ell-L_{a}-M L+s-S_{a}-M S} \sqrt{(2 L+1)(2 S+1)}\left(\begin{array}{ccc}
L_{a} & \ell & L \\
M L_{a} & m & -M L
\end{array}\right)\left(\begin{array}{ccc}
S_{a} & s & S \\
M L_{a} & m_{s} & -M S
\end{array}\right)
\end{aligned}
$$$$
\left|\mathrm{n}_{\mathrm{a}} \mathrm{L}_{\mathrm{a}} \mathrm{S}_{\mathrm{a}} \ell, \mathrm{LMLSMS}\right\rangle
$$

A derivation of equation (13) based on the use of close-coupling final state wave Iunctions has been presented by Burke (1968). However, it is evident from the derivation that the validity of the result is not restricted by the close-coupling approximation.

After substituting the partial wave expansion (12) into equation (3) and using the Wigner-Eckart theorem, the matrix elements OI $\underline{S}^{(\mathbb{N})}$ are evaluated in the form

$$
\begin{aligned}
& \left\langle M_{\mathrm{a}} \mathrm{m}_{\mathrm{s}}\left|\underline{S}^{(\mathrm{N})}\right| \mathrm{M}_{\mathrm{i}} \lambda_{1} \ldots \lambda_{\mathrm{N}}\right\rangle=\sum_{\mathrm{K} N} \sum_{\ell_{\mathrm{m}}} \sum_{J \mathbf{M}} \sum_{J^{(1)} M^{(1)} \ldots J^{(\mathbb{N}-1)} M^{\left(N^{-}-1\right)}} \\
& (-1)^{S-J_{a}-N+K-\ell+J-2 M} \sqrt{(2 K+1)(2 J+1)} \\
& \left(\begin{array}{ccc}
J_{a} & s & K \\
M_{a} & m_{s} & -N
\end{array}\right)\left(\begin{array}{ccc}
l & K & J \\
m & N & -M
\end{array}\right)\left(\begin{array}{ccc}
J & 1 & J^{(N-1)} \\
-M & \lambda_{N} & M^{(N-1)}
\end{array}\right) \\
& {\left[\prod_{v=1}^{N-2}(-1)^{J^{(v+1)}-M^{(v+1)}}\left(\begin{array}{ccc}
J^{(v+1)} & 1 & J^{(v)} \\
-M^{(v+1)} & \lambda_{v+1} & M^{(v)}
\end{array}\right)\right](-1)^{J^{(1)}-M^{(1)}}\left(\begin{array}{ccc}
J^{(1)} & 1 & J_{i} \\
-M^{(1)} & \lambda_{1} & M_{i}
\end{array}\right)}
\end{aligned}
$$

$M(u) Y_{\ell_{m}}\left(\hat{k}_{a}\right)$, 
where the symbol $u$ is used to denote the channel angular momenta $\mathrm{K} \ell \mathrm{J} \mathrm{J}^{(1)} \ldots \mathrm{J}^{(\mathrm{N}-1)}$, and the quantities $\mathrm{M}(\mathrm{u})$ are deIined in terms of the reduced electric-dipole matrix elements by

$M(u)=2 \pi \sqrt{\alpha \omega} e^{i(\sigma \ell-\ell \pi / 2)}$

$\sum_{n^{(1)} \ldots n^{(N-1)}} \frac{\left(n_{a} J_{a} K l, J\left\|\vec{Q}^{(1)}\right\| n_{\left.n^{(N-1)} J^{(N-1)}\right)}^{E\left(n^{(N-1)} J^{(N-1)}\right)-E_{\left(n_{i} J_{i}\right)}-(N-1) \omega}\right.}{(N-1)}$

$\left[\prod_{v=1}^{N-2} \frac{\left(n^{(v+1)} J^{(v+1)}\left\|\vec{Q}^{(1)}\right\| n^{(v)} J^{(v)}\right)}{E\left(n^{(v)} J^{(v)}\right)-E_{\left(n_{i} J_{i}\right)}-v \omega}\right]$

$\left(n^{(1)} J^{(1)}\left\|\vec{Q}^{(1)}\right\| n_{i} J_{i}\right)$

Perturbation theory has been used to obtain an explicit expression for the dynamical quantities $M(u)$ which may be evaluated numerically with approximate atomic wave Iunctions. However, it will become evident that only the de pendence on the projection quantum numbers given by equation (14) will be assumed in the subsequent analysis. Therefore, the question of the validity of the perturbation expression (15) will not be relevant to the derivation presented in this section. After the expansions (10) and (14) are substituted into equation (6), the relation between the spherical tensor components of $\rho_{2}^{(\mathrm{N})}, \rho_{\mathbf{i}}$, and $\rho_{r}$ is found to be expressible in the form

$$
\begin{aligned}
& \rho_{2}^{(N)}\left(K_{a} K_{s} L, N_{a} N_{s} M\right)=\sqrt{\frac{\left(2 K_{a}+1\right)\left(2 K_{s}+1\right)(2 L+1)}{4 \pi}} \\
& \sum_{K_{i} N_{i}} \sum_{K_{r}^{(1)} N_{r}^{(1)} \ldots K_{r}^{(N)} N_{r}^{(N)}} \sqrt{\left(2 K_{i}+1\right)} \rho_{i}\left(K_{i}, N_{i}\right)
\end{aligned}
$$


$\sqrt{\left(2 \mathrm{~K}_{\mathrm{r}}^{(1)}+1\right)} \rho_{\mathrm{r}}\left(\mathrm{K}_{\mathrm{r}}^{(1)}, \mathrm{N}_{\mathrm{r}}^{(1)}\right) \cdots \sqrt{\left(2 \mathrm{~K}_{\mathrm{r}}^{(\mathrm{N})}+1\right)} \rho_{\mathrm{r}}\left(\mathrm{K}_{\mathrm{r}}^{(\mathrm{N})}, \mathrm{N}_{\mathrm{r}}^{(\mathrm{N})}\right)$

$\sum_{u_{1}} \sum_{u_{2}} B\left(u_{1}, u_{2}\right) M\left(u_{1}\right) M\left(u_{2}\right)^{*}$

Using the appropriate vector coupling identities, the expression obtained for the recoupling coeficicients $B\left(u_{1}, u_{2}\right)$ may be reduced to the convenient form

$$
\begin{aligned}
& \mathrm{B}\left(\mathrm{u}_{1}, \mathrm{u}_{2}\right)=\sqrt{\left(2 \mathrm{~K}_{1}+1\right)\left(2 \mathrm{~K}_{2}+1\right)\left(2 \ell_{1}+1\right)\left(2 \ell_{2}+1\right)\left(2 \mathrm{~J}_{1}+1\right)\left(2 \mathrm{~J}_{2}+1\right)} \\
& \left(\begin{array}{lll}
\ell_{1} & \ell_{2} & \mathrm{~L} \\
0 & 0 & 0
\end{array}\right) \sum_{\mathrm{K}_{12} \mathrm{~N}_{12}} \sum_{\mathrm{J}_{12} \mathrm{M}_{12}} \sum_{\mathrm{J}_{12}{ }^{(1)} \mathrm{M}_{12}^{(1)} \cdots \mathrm{J}_{12}^{(\mathrm{N}-1)} \mathrm{M}_{12}(\mathrm{~N}-1)}\left(2 \mathrm{~K}_{12}+1\right)\left(2 \mathrm{~J}_{12}+1\right)(-1)^{\ell_{2}+\mathrm{K}_{2}-\mathrm{K}_{1}+\mathrm{K}_{12}-\mathrm{N}_{12}}
\end{aligned}
$$$$
\left(\begin{array}{ccc}
\mathrm{K}_{\mathrm{a}} & \mathrm{K}_{\mathrm{s}} & \mathrm{K}_{12} \\
\mathrm{~N}_{\mathrm{a}} & \mathrm{N}_{\mathrm{s}} & -\mathrm{N}_{12}
\end{array}\right)\left(\begin{array}{ccc}
\mathrm{K}_{12} & \mathrm{~L} & \mathrm{~J}_{12} \\
\mathrm{~N}_{12} & \mathrm{M} & -\mathrm{M}_{12}
\end{array}\right)\left(\begin{array}{ccc}
\mathrm{J}_{12} & \mathrm{~K}_{\mathrm{r}}^{(\mathrm{N})} & \mathrm{J}_{12}^{(\mathrm{N}-1)} \\
-\mathrm{M}_{12} & \mathrm{~N}_{\mathrm{r}}^{(\mathrm{N})} & \mathrm{M}_{12}^{(\mathrm{N}-1)}
\end{array}\right)
$$

$\left\{\begin{array}{lll}J_{a} & s & K_{2} \\ J_{a} & s & K_{1} \\ K_{a} & K_{s} & K_{12}\end{array}\right\}\left\{\begin{array}{lll}K_{2} & l_{2} & J_{2} \\ K_{1} & \ell_{1} & J_{1} \\ K_{12} & L & J_{12}\end{array}\right\}\left\{\begin{array}{ccc}J_{2} & 1 & J_{2}^{(N-1)} \\ J_{1} & 1 & J_{1}^{(N-1)} \\ J_{12} & K_{r}^{(N)} & J_{12}^{(N-1)}\end{array}\right\}$

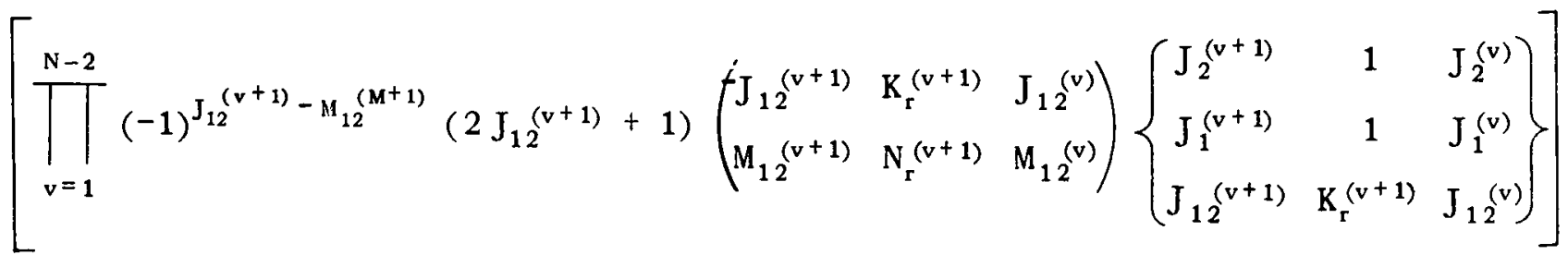

$(-1)^{\mathrm{J}_{12}^{(1)}-\mathrm{M}_{12}^{(1)}}\left(2 \mathrm{~J}_{12}{ }^{(1)}+1\right)\left(\begin{array}{ccc}\mathrm{J}_{12}^{(1)} & \mathrm{K}_{\mathrm{r}}^{(1)} & \mathrm{K}_{\mathrm{i}} \\ -\mathrm{M}_{12}{ }^{(1)} & \mathrm{N}_{\mathrm{r}}^{(1)} & \mathrm{N}_{\mathrm{i}}\end{array}\right)\left\{\begin{array}{lll}\mathrm{J}_{2}^{(1)} & 1 & \mathrm{~J}_{\mathrm{i}} \\ \mathrm{J}_{1}^{(1)} & 1 & \mathrm{~J}_{\mathrm{i}} \\ \mathrm{J}_{12}^{(1)} & \mathrm{K}_{\mathrm{r}}^{(1)} & \mathrm{K}_{\mathrm{i}}\end{array}\right\}$ 
In equations (14), (15), and (17) the terms inside the square brackets are to be omitted for the case of $\mathbb{N}=2$.

From the selection rule that $\ell_{1}+\ell_{2}+\mathrm{L}$ must be an even integer and the requirement that the values of $l_{1}$ and $l_{2}$ which are associated with a given ionic state must correspond to the same parity, it follows that only even values of $I$ are involved in the spherical harmonic expansion of equation (11). Using the abbreviation $I=J_{a}+s+J_{i}$, the maximum allowed value of $L$ is given by

$$
L_{\max }=\left\{\begin{array}{ll}
2(I+N) & \text { for integral values of } I \\
2(I+N)-1 & \text { for half-integral values of } I
\end{array}\right\}
$$

It should be clear that the maximum value of $\mathrm{L}$ may not be realized unless the target atom is polarized and the polarizations of the residual ion and the ejected electron are observed.

In the case of the IS-representation, the density matrices ${\underline{\rho_{i}}}_{\mathbf{i}}$ and $\rho_{2}^{(\mathbb{N})}$ may be expressed as direct products of density matrices referring to either the orbital or the spin angular momenta. The polarization state of the target atom is now represented by the spherical tensor components $\rho_{\mathrm{L}_{1}}\left(\mathrm{KL}_{\mathrm{i}}, \mathrm{NL}_{\mathrm{i}}\right)$ and $\rho_{\mathrm{S}_{\mathrm{i}}}\left(\mathrm{K} \mathrm{S}_{\mathrm{i}}, \mathrm{NS}_{\mathrm{i}}\right)$. The final state polarization density matrix is now expanded in a form which is analogous to equation (II) but with the separated components $\rho_{L_{a}} l^{(N)}\left(K L_{a} L, N L_{a} M\right)$ and $\left.\rho_{\mathrm{S}_{\mathrm{a}}}{ }_{\mathrm{N}}{ }^{\mathrm{N}}\right)\left(\mathrm{KS} \mathrm{S}_{\mathrm{a}} \mathrm{K}_{\mathrm{s}}, \mathrm{NS}_{\mathrm{a}} \mathrm{N}_{\mathrm{s}}\right)$. For the case of $\mathrm{N}=2$, the recoupling coefficient which occurs in the analogue or equation (16) may be expressed in the form 


$$
\begin{aligned}
& \mathrm{B}\left(\ell_{1} \mathrm{~L}_{1} \mathrm{~L}_{1}^{(1)}, \ell_{2} \mathrm{~L}_{2} \mathrm{~L}_{2}^{(1)}\right)=\left(2 \mathrm{~S}_{\mathrm{i}}+1\right) \sqrt{\left(2 \ell_{1}+1\right)\left(2 \ell_{2}+1\right)\left(2 \mathrm{~L}_{1}+1\right)\left(2 \mathrm{~L}_{2}+1\right)} \\
& \left(\begin{array}{lll}
\ell_{1} & \ell_{2} & \mathrm{~L} \\
0 & 0 & 0
\end{array}\right) \sum_{\mathrm{L}_{12} \mathrm{M}_{12}} \sum_{\mathrm{L}_{12}^{(1)} \mathrm{M}_{12}^{(1)}}\left(2 \mathrm{~L}_{12}+1\right)\left(2 \mathrm{~L}_{12}^{(1)}+1\right)(-1)^{\ell_{2}+K S_{1}-\mathrm{NS}_{1}+\mathrm{L}_{12}{ }_{12}^{(1)}-\mathrm{M}_{12}{ }^{(1)}} \\
& \left(\begin{array}{ccc}
K_{\mathrm{a}} & \mathrm{K}_{\mathrm{s}} & \mathrm{KS}_{\mathrm{i}} \\
\mathrm{N} \mathrm{S}_{\mathrm{a}} & \mathrm{N}_{\mathrm{s}} & -\mathrm{NS}_{\mathrm{i}}
\end{array}\right)\left(\begin{array}{ccc}
\mathrm{KL}_{\mathrm{a}} & \mathrm{L} & \mathrm{L}_{12} \\
\mathrm{NL}_{\mathrm{a}} & \mathrm{M} & -\mathrm{ML}_{12}
\end{array}\right)\left(\begin{array}{ccc}
\mathrm{L}_{12} & \mathrm{~K}_{\mathrm{r}}^{(2)} & \mathrm{L}_{12}^{(1)} \\
-\mathrm{ML}_{12} & \mathrm{~N}_{\mathrm{r}}^{(2)} & \mathrm{ML}_{12}^{(1)}
\end{array}\right)\left(\begin{array}{ccc}
\mathrm{L}_{12}(1) & \mathrm{K}_{\mathrm{r}}^{(1)} & \mathrm{KL}_{\mathrm{i}} \\
-\mathrm{ML}_{12}(1) & \mathrm{N}_{\mathrm{r}}^{(1)} & \mathrm{NL}_{\mathrm{i}}
\end{array}\right) \\
& \left\{\begin{array}{ccc}
\mathrm{S}_{\mathrm{a}} & \mathrm{S} & \mathrm{S}_{\mathrm{i}} \\
\mathrm{S}_{\mathrm{a}} & \mathrm{S} & \mathrm{S}_{\mathrm{i}} \\
\mathrm{KS}_{\mathrm{a}} & \mathrm{K}_{\mathrm{s}} & \mathrm{KS}_{\mathrm{i}}
\end{array}\right\}\left\{\begin{array}{ccc}
\mathrm{L}_{\mathrm{a}} & \mathrm{l}_{2} & \mathrm{~L}_{2} \\
\mathrm{~L}_{\mathrm{a}} & \ell_{1} & \mathrm{~L}_{1} \\
\mathrm{KL}_{\mathrm{a}} & \mathrm{L} & \mathrm{L}_{12}
\end{array}\right\}\left\{\begin{array}{ccc}
\mathrm{L}_{2} & 1 & \mathrm{~L}_{2}^{(1)} \\
\mathrm{L}_{1} & 1 & \mathrm{~L}_{1}^{(1)} \\
\mathrm{L}_{12} & \mathrm{~K}_{\mathrm{r}}^{(2)} & \mathrm{L}_{12}(1)
\end{array}\right\}\left\{\begin{array}{ccc}
\mathrm{L}_{2}^{(1)} & 1 & \mathrm{~L}_{\mathrm{i}} \\
\mathrm{L}_{1}^{(1)} & 1 & \mathrm{~L}_{\mathrm{i}} \\
\mathrm{L}_{12}(1) & \mathrm{K}_{\mathrm{r}}^{(1)} & \mathrm{KL}_{\mathrm{i}}
\end{array}\right\}
\end{aligned}
$$

which may also be written as a product of factors containing either spin or orbital angular momenta. The generalization of equation (19) for $\mathbb{N}>2$ becomes apparent when the comparison with equation (17) is made.

\section{Multiphoton Photoelectron Spectroscopy Measurements}

When the ejected electron and the residual ion polarizations are not measured, the angular distribution of the ejected electrons is given by the partial differential cross section

$$
\sigma_{a}^{(N)}\left(\hat{k}_{a}\right)=\operatorname{tr}\left(\rho_{2}^{(N)}\right)
$$


From equation (11) it follows that this differential cross section may be expressed in the form

$\sigma_{a}^{(N)}\left(\hat{k}_{a}\right)=\sum_{L M} \sqrt{\left(2 J_{a}+1\right)(2 s+1)} \rho_{2}^{(N)}(00 L, 00 M) Y_{L M}\left(\hat{k}_{a}\right)$,

where the maximum value of the even integer $I$ is $2\left(J_{i}+N\right)$ for integral values of $J_{i}$ and $2\left(J_{i}+N\right)-1$ for hall-integral values of $J_{i} \cdot$

For an unpolarized target atom the coordinate system can be conveniently chosen so that the form of the partial differential cross section (21) reduces to

$$
\sigma_{a}^{(N)}\left(\hat{k}_{a}\right)=\frac{\sigma_{a}^{(N)}}{4 \pi}\left[1+\sum_{L=2}^{2 N} \beta_{a L}{ }^{(N)} P_{L}(\cos \theta)\right],
$$

where $\sigma_{a}^{(\mathbb{N})}$ is the total cross section for the ionic state a, and the asymmetry parameters which occur in the Legendre polynomial expansion are derined by

$$
\sigma_{a}^{(N)} \beta_{a \mathrm{~L}}^{(N)}=\sqrt{4 \pi\left(2 \mathrm{~J}_{\mathrm{a}}+1\right)(2 \mathrm{~s}+1)(2 \mathrm{~L}+1)} \rho_{2}^{(\mathrm{N})}(00 \mathrm{~L}, 00 \mathrm{M})
$$

If the incident radiation is unpolarized or circularly polarized, the $\mathrm{z}$-axis is to be taken as the propagation direction or the incident photons. For linearly polarized light the appropriate choice of the z-axis is the photon polarization direction. The electric-dipole photon tensor components for these polarization states are given in Table I for later reference. 
As an illustration of the use of the theoretical Iramework developed in the previous section, it is of interest to consider twophoton ionization for the usual experimental condition of randomly oriented target atoms. The cross section $\sigma_{a}^{(2)}$ is given in the J-representation by

$$
\begin{aligned}
& \sigma_{\mathrm{a}}^{(2)}=\frac{1}{\left(2 \mathrm{~J}_{\mathrm{i}}+1\right)} \sum_{\mathrm{K}_{\mathrm{r}}} \sum_{\mathrm{K} \ell \mathrm{J}} \sum_{\mathrm{J}_{1}^{(1)} \mathrm{J}_{2}^{(1)}}(-1)^{\mathrm{K}_{\mathrm{r}}+\mathrm{J}_{\mathrm{i}}+\mathrm{J}+\mathrm{J}_{1}^{(1)}+\mathrm{J}_{2}^{(1)}} \\
& {\left[\rho_{\mathrm{r}}\left(\mathrm{K}_{\mathrm{r}}, 0\right)\right]^{2}\left\{\begin{array}{ccc}
1 & 1 & \mathrm{~K}_{\mathrm{r}} \\
\mathrm{J}_{2}^{(1)} & \mathrm{J}_{1}^{(1)} & \mathrm{J}_{\mathrm{i}}
\end{array}\right\}\left\{\begin{array}{ccc}
1 & 1 & \mathrm{~K}_{\mathrm{r}} \\
\mathrm{J}_{2}^{(1)} & \mathrm{J}_{1}^{(1)} & \mathrm{J}
\end{array}\right\}}
\end{aligned}
$$

$M\left(K \ell J_{1}^{(1)}\right) M\left(K \ell J_{2}^{(1)}\right)^{*}$

and in the LS-representation by

$$
\begin{gathered}
\sigma_{\mathrm{a}}^{(2)}=\frac{1}{\left(2 \mathrm{~L}_{\mathrm{i}}+1\right)} \sum_{\mathrm{K}_{\mathrm{r}}} \sum_{\ell_{1} \mathrm{~L}_{1}} \sum_{\mathrm{L}_{1}^{(1)} \mathrm{L}_{2}^{(1)}}(-1)^{\mathrm{K}_{\mathrm{r}}+\mathrm{L}_{\mathrm{i}}+\mathrm{L}_{1}} \\
{\left[\rho_{\mathrm{r}}\left(\mathrm{K}_{\mathrm{r}}, 0\right)\right]^{2}\left\{\begin{array}{ccc}
1 & 1 & \mathrm{~K}_{\mathrm{r}} \\
\mathrm{L}_{2}^{(1)} & \mathrm{L}_{1}^{(1)} & \mathrm{L}_{\mathrm{i}}
\end{array}\right\}\left\{\begin{array}{ccc}
1 & 1 & \mathrm{~K}_{\mathrm{r}} \\
\mathrm{L}_{2}^{(1)} & \mathrm{L}_{1}^{(1)} & \mathrm{L}_{1}
\end{array}\right\}}
\end{gathered}
$$

$M\left(\ell_{1} L_{1} L_{1}^{(1)}\right) M\left(\ell_{1} L_{1} L_{2}^{(1)}\right)^{*}$

From the photon tensor components given in Table 1 , it is clear that the total cross section $\sigma_{\mathrm{a}}^{(2)}$ depends on the polarization state of the incident radiation. In contrast, the total cross-section for singlephoton ionization of an unpolarized target atom involves only the tensor component $\rho_{\mathrm{r}}(0,0)$, which is independent of the polarization state. Equations (24) and (25) illustrate the nonlinear dependence on the photon polarization discussed by Lambropoulos (1972a). 
The angular distribution of photoelectrons resulting from twophoton ionization is characterized by the two asymmetry parameters $\beta_{\mathrm{a} 2}{ }^{(2)}$ and $\beta_{\mathrm{a} 4}{ }^{(2)}$. In the J-representation these two asymmetry parameters are given by

$$
\begin{aligned}
& \beta_{\mathrm{aL}}^{(2)}=\frac{(2 \mathrm{~L}+1)}{\sigma_{\mathrm{a}}^{(2)}\left(2 \mathrm{~J}_{\mathrm{i}}+1\right)} \sum_{\mathrm{K}_{\mathrm{r}}^{(1)}} \sum_{\mathrm{K}_{\mathrm{r}}^{(2)}} \sqrt{\left(2 \mathrm{~K}_{\mathrm{r}}^{(1)}+1\right)\left(2 \mathrm{~K}_{\mathrm{r}}^{(2)}+1\right)} \rho_{\mathrm{r}}\left(\mathrm{K}_{\mathrm{r}}^{(1)}, 0\right) \rho_{\mathrm{r}}\left(\mathrm{K}_{\mathrm{r}}^{(2)}, 0\right) \\
& \sum_{\mathrm{K}_{1} \ell_{1} \mathrm{~J}_{1} \mathrm{~J}_{1}^{(1)}} \sum_{\mathrm{K}_{2} \ell_{2} \mathrm{~J}_{2} \mathrm{~J}_{2}^{(1)}} \delta\left(\mathrm{K}_{1}, \mathrm{~K}_{2}\right)(-1)^{\mathrm{J}_{2}+\mathrm{K}_{2}+\mathrm{J}_{\mathrm{i}}+\mathrm{J}_{1}^{(1)}+1+\mathrm{K}_{\mathrm{r}}^{(1)}} \\
& \sqrt{\left(2 \ell_{1}+1\right)\left(2 \ell_{2}+1\right)\left(2 \mathrm{~J}_{1}+1\right)\left(2 \mathrm{~J}_{2}+1\right)}\left(\begin{array}{ccc}
\ell_{1} & \ell_{2} & \mathrm{~L} \\
0 & 0 & 0
\end{array}\right)\left(\begin{array}{ccc}
\mathrm{K}_{\mathrm{r}}^{(1)} & \mathrm{K}_{\mathrm{r}}^{(2)} & \mathrm{L} \\
0 & 0 & 0
\end{array}\right) \\
& \left\{\begin{array}{lll}
\ell_{1} & \ell_{2} & \mathrm{~L} \\
\mathrm{~J}_{2} & \mathrm{~J}_{1} & \mathrm{~K}_{1}
\end{array}\right\}\left\{\begin{array}{ccc}
1 & 1 & \mathrm{~K}_{\mathrm{r}}^{(1)} \\
\mathrm{J}_{2}^{(1)} & \mathrm{J}_{1}^{(1)} & \mathrm{J}_{\mathrm{i}}
\end{array}\right\}\left\{\begin{array}{ccc}
\mathrm{J}_{2} & 1 & \mathrm{~J}_{2}^{(1)} \\
\mathrm{J}_{1} & 1 & \mathrm{~J}_{1}^{(1)} \\
\mathrm{L} & \mathrm{K}_{\mathrm{r}}^{(2)} & \mathrm{K}_{\mathrm{r}}^{(1)}
\end{array}\right\} \mathrm{M}\left(\mathrm{K}_{1} \ell_{1} \mathrm{~J}_{1} \mathrm{~J}_{1}^{(1)}\right) \mathrm{M}\left(\mathrm{K}_{2} \ell_{2} \mathrm{~J}_{2} \mathrm{~J}_{2}^{(1)}\right)^{*}
\end{aligned}
$$

The corresponding expression for $\beta_{\mathrm{aL}}{ }^{(2)}$ in the LS-representation is

$$
\begin{aligned}
& \beta_{a L}^{(2)}=\frac{(2 \mathrm{~L}+1)}{\sigma_{a}^{(2)}\left(2 \mathrm{~L}_{\mathrm{i}}+1\right)} \sum_{\mathrm{K}_{\mathrm{r}}^{(1)}} \sum_{\mathrm{K}_{\mathrm{r}}^{(2)}} \sqrt{\left(2 \mathrm{~K}_{\mathrm{r}}^{(1)}+1\right)\left(2 \mathrm{~K}_{\mathrm{r}}^{(2)}+1\right)} \rho_{\mathrm{r}}\left(\mathrm{K}_{\mathrm{r}}^{(1)}, 0\right) \rho_{\mathrm{r}}\left(\mathrm{K}_{\mathrm{r}}^{(2)}, 0\right) \\
& \sum_{\ell_{1} \mathrm{~L}_{1} \mathrm{~L}_{1}^{(1)}} \sum_{\ell_{2} \mathrm{~L}_{2} \mathrm{~L}_{2}^{(1)}}(-1)^{\mathrm{L}_{2}+\mathrm{L}_{\mathrm{a}}+\mathrm{L}_{1}+\mathrm{L}_{1}^{(1)}+1+\mathrm{K}_{\mathrm{r}}^{(1)}} \\
& \sqrt{\left(2 \ell_{1}+1\right)\left(2 \ell_{2}+1\right)\left(2 \mathrm{~L}_{1}+1\right)\left(2 \mathrm{~L}_{2}+1\right)}\left(\begin{array}{ccc}
\ell_{1} & \ell_{2} & \mathrm{~L} \\
0 & 0 & 0
\end{array}\right)\left(\begin{array}{ccc}
\mathrm{K}_{\mathrm{r}}^{(1)} & \mathrm{K}_{\mathrm{r}}^{(2)} & \mathrm{L} \\
0 & 0 & 0
\end{array}\right) \\
& \left\{\begin{array}{lll}
\ell_{1} & \ell_{2} & \mathrm{~L} \\
\mathrm{~L}_{2} & \mathrm{~L}_{1} & \mathrm{~L}_{\mathrm{a}}
\end{array}\right\}\left\{\begin{array}{ccc}
1 & 1 & \mathrm{~K}_{\mathrm{r}}^{(1)} \\
\mathrm{L}_{2}^{(1)} & \mathrm{L}_{1}^{(1)} & \mathrm{L}_{\mathrm{i}}
\end{array}\right\}\left\{\begin{array}{ccc}
\mathrm{L}_{2} & 1 & \mathrm{~L}_{2}^{(1)} \\
\mathrm{L}_{1} & 1 & \mathrm{~L}_{1}^{(1)} \\
\mathrm{L}^{(1)} & \mathrm{K}_{\mathrm{r}}^{(2)} & \mathrm{K}_{\mathrm{r}}^{(1)}
\end{array}\right\} M\left(\ell_{1} \mathrm{~L}_{1} \mathrm{~L}_{1}^{(1)}\right) M\left(\ell_{2} \mathrm{~L}_{2} \mathrm{~L}_{2}^{(1)}\right)^{*} \quad(27)
\end{aligned}
$$


The requirement that the angular distribution given by equation (22) with $N=2$ be a non-negative function of $\theta$ implies that the values of the asymmetry parameters $\beta_{\mathrm{a} 2}{ }^{(2)}$ and $\beta_{\mathrm{a} 4}{ }^{(2)}$ are restricted by the inequalities

$$
\begin{gathered}
\beta_{\mathrm{a} 2}^{(2)}+\beta_{\mathrm{a} 4}{ }^{(2)} \geq-1 \\
4 \beta_{\mathrm{a} 2}^{(2)}-3 \beta_{\mathrm{a} 4}^{(2)} \leq 8
\end{gathered}
$$

The form of the photoelectron angular distribution resulting from twophoton ionization has also been discussed by Lambropoulos and Berry (1972), who have derived an explicit expression for the asymmetry parameters using a density matrix formalism similar to the formalism employed in section 3. However, their treatment is restricted to the case where there is only one value of the intermediate angular momentum $I^{(1)}$.

The case of two-photon ionization from an s-state of the target atom is of particular experimental interest. In the LS-representation, the allowed virtual intermediate states are the P-states. The triangle conditions imply that the allowed values of the final state total angular momentum $I_{I}$ are $0, I$ and 2 . In the case of circularly polarized light, however, angular momentum coniservation implies that only the D.partial wave component will be excited. This also follows from equations (25) and (27) which now reduce to

$$
\sigma_{\mathrm{a}}^{(2)}=\frac{1}{3} \sum_{\mathrm{K}_{\mathrm{r}}} \sum_{\ell_{1}}\left[\rho_{\mathrm{r}}\left(\mathrm{K}_{\mathrm{r}}, 0\right)\right]^{2}\left\{\begin{array}{lll}
1 & 1 & \mathrm{~K}_{\mathrm{r}} \\
1 & 1 & 2
\end{array}\right\}\left|\mathrm{M}\left(\ell_{1}, 2,1\right)\right|^{2}
$$

and 


$$
\begin{aligned}
& \beta_{\mathrm{aL}}^{(2)}=\frac{5(2 \mathrm{~L}+1)(-1)^{\mathrm{L}_{\mathrm{a}}}}{3 \sigma_{\mathrm{a}}^{(2)}} \sum_{\mathrm{K}_{\mathrm{r}}^{(1)}} \sum_{\mathrm{K}_{\mathrm{r}}^{(2)}} \sqrt{\left(2 \mathrm{~K}_{\mathrm{r}}^{(1)}+1\right)\left(2 \mathrm{~K}_{\mathrm{r}}^{(2)}+1\right)} \rho_{\mathrm{r}}\left(\mathrm{K}_{\mathrm{r}}^{(1)}, 0\right) \rho_{\mathrm{r}}\left(\mathrm{K}_{\mathrm{r}}^{(2)}, 0\right) \\
& \sum_{\ell_{1}} \sum_{\ell_{2}} \sqrt{\left(2 \ell_{1}+1\right)\left(2 \ell_{2}+1\right)}\left(\begin{array}{ccc}
\ell_{1} & \ell_{2} & \mathrm{~L} \\
0 & 0 & 0
\end{array}\right)\left(\begin{array}{ccc}
\mathrm{K}_{\mathrm{r}}^{(1)} & \mathrm{K}_{\mathrm{r}}^{(2)} & \mathrm{L} \\
0 & 0 & 0
\end{array}\right) \\
& \left\{\begin{array}{lll}
\ell_{1} & \ell_{2} & \mathrm{~L} \\
2 & 2 & \mathrm{~L}_{\mathrm{a}}
\end{array}\right\}\left\{\begin{array}{ccc}
2 & 1 & 1 \\
2 & 1 & 1 \\
\mathrm{~L} & \mathrm{~K}_{\mathrm{r}}^{(1)} & \mathrm{K}_{\mathrm{r}}^{(2)}
\end{array}\right\} \quad M\left(\ell_{1}, 2,1\right) \mathrm{M}\left(\ell_{2}, 2,1\right)^{*},
\end{aligned}
$$

where the photon tensor components for the case of circular polarization are to be used. Lambropoulos (1972) has given particular emphasis to the fact that only the dynamical quantities $M$ corresponding to the $I=\mathbb{N}$ partial wave component of the final state occur in the expression for the differential $\mathbb{N}$-photon ionization cross section for circularly polarized light. A Iurther significant Ieature is that equations (30) and (31) also apply to single-photon ionization from an aligned P-state provided that the quantities $M$ are suitably reinterpreted. This result is in agreement with the explanation of the photon polarization eifect given by Fox et al. (1971).

The component of the photoelectron spin polarization in the direction of the incident radiation, which is produced by $\mathrm{N}$-photon ionization of a randomly oriented atom, may be expressed in the form

$$
\mathrm{P}_{\mathrm{a}}^{(\mathrm{N})}\left(\hat{\mathrm{k}}_{\mathrm{a}}\right)=\frac{\sigma_{\mathrm{a}}^{(\mathrm{N})}}{4 \pi \sigma_{\mathrm{a}}^{(\mathrm{N})}\left(\hat{\mathrm{k}}_{\mathrm{a}}\right)}\left[\mathrm{P}_{\mathrm{a}}^{(\mathrm{N})}+\sum_{\mathrm{L}=2}^{2 \mathrm{~N}} \gamma_{\mathrm{aL}}{ }^{(\mathrm{N})} \mathrm{P}_{\mathrm{L}}(\cos \theta)\right],
$$

where it is assumed that the polarization state of the residual ion is not detected and $\theta$ measures the angle between the photoelectron direction and the direction of the incident radiation. The total 
polarization $\mathrm{P}_{\mathrm{a}}^{(\mathrm{N})}$ and the asymmetry parameters $\gamma_{\mathrm{aL}}{ }^{(\mathrm{N})}$ are given in terms of the tensor components of the final state density matrix by the relations

$$
\sigma_{\mathrm{a}}^{(\mathrm{N})} \mathrm{P}_{\mathrm{a}}^{(\mathrm{N})}=\sqrt{4 \pi\left(2 \mathrm{~J}_{\mathrm{a}}+1\right)(2 \mathrm{~s}+1)} \rho_{2}^{(\mathrm{N})}(010,000)
$$

and

$$
\sigma_{\mathrm{a}}^{(\mathrm{N})} \gamma_{\mathrm{aL}}^{(\mathrm{N})}=\sqrt{4 \pi\left(2 \mathrm{~J}_{\mathrm{a}}+1\right)(2 \mathrm{~s}+1)(2 \mathrm{~L}+1)} \rho_{2}^{(\mathrm{N})}(01 \mathrm{~L}, 000)
$$

Since Fano (1969) has shown that the spin polarization of photoelectrons ejected from randomly oriented alkali atoms is determined by the direct action of the spin-orbit force on the continuum states, the use of the J-representation is required Ior the correct determination of this polarization. From equation (17), it is evident that the photoelectron spin polarization is nonzero only when the following conditions are satisfied

$$
\begin{aligned}
& \mathrm{J}_{12}^{(1)} \quad=\mathrm{K}_{\mathrm{r}}^{(1)} \\
& \mathrm{J}_{12}(\mathrm{v}+1)+\mathrm{K}_{\mathrm{r}}^{(\mathrm{v}+1)}+\mathrm{J}_{12}(\mathrm{v})=\text { even integer, } \mathrm{v}=1, \mathrm{~N}-2 \\
& \mathrm{~J}_{12}+\mathrm{K}_{\mathrm{r}}^{(\mathrm{N})}+\mathrm{J}_{12}(\mathrm{~N}-1)=\text { even integer } \\
& \mathrm{J}_{12}+1=\text { even integer }
\end{aligned}
$$

Using the properties of the photon tensor components given in Table I, it is found that in the electric-dipole approximation the photoelectron spin polarization produced by multiphoton ionization of any order, like the analogous polarization resulting from single-photon ionization, is determined only by the circularly polarized component of the incident radiation. 


\section{Acknowledgments}

I am greatly indebted to $\mathrm{Dr}$. J. Mizuno for helpiul discussions which motivated this investigation.

This research has been made possible by a National Research Council Resident Research Associateship. 
$\underline{\text { ReIerences }}$

Bebb H G and Gold A 1966 Phys. Rev. I43, I-24

Blatt J M and Biedenharn I C 1952 Rev. Mod. 24 258-72

Breit $G$ and Bethe H A 1954 Phys. Rev 23 888-90

Burke P G 1968 The Physics of Electronic and Atomic Collisions (Boulder, Colorado: The Joint Institute for Laboratory Astrophysics) $128-44$

Faisal F H M 1972 J. Phys. B: Atom. Molec. Phys. 2 Ir 36

Fano U 1957 Rev. Mod. Phys. 29 74-93

Fano U 1969 Phys. Rev. 178 131-36

Fano U and Racah G 1959 Irreducible Tensorial Sets (New York: Academic)

Fox R A, Kogan R M and Robinson E J 1971 Phys. Rev. Let. 26 1416-7

Happer W 1968 Annals of Physics 48 579-91.

Jacobs V L 1972 J. Phys. B: Atom. Molec. Phys. 2 2257-2271

Kogan R M, Fox R A, Burnham G T and Robinson E J 1971 Bull. Amer. Phys. Soc. 161411

Lambropoulos M and Berry S R 1972 Bull. Amer. Phys. Soc. 17371

Lambropoulos P 1972a Phys. Rev. Lett. 28 585-7

Lambropoulos P 1972b Phys. Rev. Lett. 29 453-5

Morton V M 1967 Proc. Phys. Soc. 22 301-10

Reiss H R 1970 Phys. Rev. Al 803-18

Shalit A de and Talmi J 1963 Nuclear Shell Theory (New York: Academic) 
Table 1

Spherical Tensor Components for Various Polarization States of Electric-Dipole Photons

\begin{tabular}{l|c|c|c}
\hline \multicolumn{1}{c|}{ polarization state } & $\rho_{r}(0,0)$ & $\rho_{r}(1,0)$ & $\rho_{r}(2,0)$ \\
\hline unpolarized & $(1 / 3)^{1 / 2}$ & 0 & $(1 / 6)^{1 / 2}$ \\
circular polarization $(\lambda= \pm 1)$ & $(1 / 3)^{1 / 2}$ & $\pm 1 / 2$ & $(1 / 6)^{1 / 2}$ \\
linear polarization & $(1 / 3)^{1 / 2}$ & 0 & $(1 / 6)^{1 / 2}$
\end{tabular}

